\title{
APPLICATION OF HM-NETWORK WITH POSITIVE AND NEGATIVE CLAIMS FOR FINDING OF MEMORY VOLUMES IN INFORMATION SYSTEMS
}

\author{
Mikhail Matalytski, Pawet Zając \\ Institute of Mathematics, Czestochowa University of Technology \\ Czestochowa, Poland \\ m.matalytski@gmail.com,pawel_zajac@vp.pl
}

Received: 7 May 2018; Accepted: 12 April 2019

\begin{abstract}
To solve the problem of determining the volume of memory of information systems (IS), it was suggested to use a stochastic model based on the use of HM (Howard-Matalytski) - a queueing network with revenues. This model allows you to take into account the dependencies of the processing times of messages (claims) on their volumes, the possibility of changing the volume of messages over time, and also the possibility of getting computer viruses into it. In such cases the processing of messages in nodes can be interrupted for some random time. Expressions are obtained for the mean (expected) values of the total message volumes in the IS nodes.
\end{abstract}

MSC 2010: 90B22, 60K25, 68M20

Keywords: HM-networks, information systems, volume of claims, positive and negative claims

\section{Introduction}

In IS, the total volume of memory is limited to a certain value, which is usually called the volume of memory [1]. When designing an IS, the main task is to find the average volume of memory in such a way as to take into account the conditions that limit the share of lost information. One of the methods for solving problems of designing IS is the use of HM-networks [2]. By IS we mean systems, objects of transformation in which information comes in portions as messages [1]. HM-networks can be used to determine the volume of buffer memory systems that are nodes of the IS processing and transmission of messages. Note that the problem under consideration is one of the main ones, for instance, when designing hubs or communication centres in data transmission networks. The model below can also be used to solve an actual problem that arose not so long ago in IS, namely, the problems of excessive buffering (i.e., determining the required volume of memory) [3]. Excessive network buffering is a phenomenon that has arisen in networks with 
packet communication when excessive buffering causes an increase in packet transit time and packet delay, and as a result, a decrease in the bandwidth of the IS.

The failure of the time dependence of the processing of messages from their volumes can lead to serious errors in finding the volumes of the buffer memory in the IS. The solution of the above problems in the general case can be based on the use of HM-networks with revenues. In such networks, the claim during the transition from one queueing system (QS) to another brings the latter some revenue (equal to the volume of this claim), and the revenue (volume) of the first QS decreases by this amount.

It should be noted that the method of finding the nonstationary state probabilities and the mean characteristics of the G-network, i.e. network with positive and negative claims operating under high load conditions (heavy-traffic regime) using the apparatus of multidimensional generating functions is described in the monograph [4], it also investigated HM-networks of this type. For the first time, the use of HM-networks for estimating memory volumes in IS was described in [5]. In [6, 7], a method for finding the mean total volume of the same type of claims in open HM-network systems with a limited number of them in the queues and bypassing the claims of the service systems has been described; in [8] - HM-network with a limited waiting time for claims in the queues of the system, and in [9] - HM-networks with unreliable service of customers in QS.

In 1991, a new class of open queueing networks with two types of claims was introduced by E. Gelenbe $[9,10]$ - "positive" and "negative" customers, they are now called G-networks. For example, in computer networks "positive" claims are tasks (programs), and "negative" claims - computer viruses [11]. This corresponds to the fact that when entering a computer network the virus destroys or harms, it infects one of the executable programs, reducing the number of active programs or requests in the system by one [12-14]. Then the virus disappears from the network, not getting any service for itself. It should be noted that the study of such networks in the stationary regime was carried out in [10-12]. It has been proven that the probability distribution of states has the product form.

G-networks are a class of stochastic models that are widely used also in the modeling of neural networks, gene regulatory networks [15-17], estimation of information systems and networks performance and modeling of energy flows in systems with renewable energy sources [18-27].

Consider an open queueing G-network with $n$ single-line QS. In QS $S_{i}$ from the outside (from the system $S_{0}$ ) an incoming flow of positive (normal) of claims intensity of $\lambda_{0 i}^{+}$and Poisson flow of negative claims intensity of $\lambda_{0 i}^{-}, i=\overline{1, n}$. All flows of claims entering the network are independent. The service time of the positive claims in the QS $S_{i}$ exponentially distributed with mean $\mu_{i}, i=\overline{1, n}$. Negative claims coming to some system of the network in which there is at least one positive message instantly destroys (destroys, removes from the network) one of them. On the assumption of an exponential distribution of service time of positive claims may not care about what kind of message is destroyed. After this, it immediately leaves 
the network itself without getting in the QS no maintenance. Thus, each QS of the network can be served by only positive claims, so in the future, when speaking about the positive claims service, usually for the sake of brevity they are simply called claims [15].

Each positive claim is sent to the QS of the $S_{i}$ with probability $p_{0 i}^{+}$, and the negative - with probability $p_{0 i}^{-}, \sum_{i=1}^{n} p_{0 i}^{+}=\sum_{i=1}^{n} p_{0 i}^{-}=1, i=\overline{1, n}$. A positive claim serviced in the QS $S_{i}$, with probability $p_{i j}^{+}$sent to the QS $S_{j}$ as a positive claim, with a probability $p_{i j}^{-}$- as a negative claim, and with probability $p_{i 0}=1-\sum_{j=1}^{n}\left(p_{i j}^{+}+p_{i j}^{-}\right)$ leaving from the network to the external environment (QS $\left.S_{0}\right), i, j=\overline{1, n}$. The state of the network meaning the vector $k(t)=(k, t)=\left(k_{1}, k_{2}, \ldots, k_{n}, t\right)$, where $k_{i}$ - the number of claims at the moment of time $t$ at the system $S_{i}, i=\overline{1, n}$.

\section{Analysis of Markov HM-networks with positive and negative claims}

We will now consider our network taking into account the change in the total volume of claims in the QS when serving positive and negative claims. Consider the case where changes in the volume of claims associated with transitions between network states are deterministic functions that depend on network states and time. Possible transitions between network states, transition probabilities and changes in the total volume of claims in the system from these transitions are indicated in Table 1. They are similar to the one in [28] for a network without negative claims.

Using the full probability formula for mathematical expectation, we obtain a system of difference-differential equations for the expected volume of claims $v_{i}(k, t)$ in the system $S_{i}$ :

$$
\begin{gathered}
\frac{d v_{i}(k, t)}{d t}=r_{i}(k)-\sum_{j=1}^{n}\left[\lambda_{0 j}^{+}+\lambda_{0 j}^{-}+\mu_{j}\right] v_{i}(k, t)+ \\
+\sum_{j=1}^{n}\left(\lambda_{0 j}^{+} v_{i}\left(k+I_{j}, t\right)+\left[\mu_{j} p_{j 0} u\left(k_{j}(t)\right)+\lambda_{0 j}^{-} u\left(k_{j}(t)\right)+\mu_{j} \sum_{\substack{c=1 \\
c \neq j}}^{n} p_{j c}^{-}\left(1-u\left(k_{c}(t)\right)\right)\right] v_{i}\left(k-I_{j}, t\right)\right]+ \\
+\lambda_{0 i}^{+} v_{i}\left(k+I_{i}, t\right)+\left[\mu_{i} p_{i 0} u\left(k_{i}(t)\right)+\lambda_{0 i}^{-} u\left(k_{i}(t)\right)+\mu_{i} \sum_{\substack{c=1 \\
c \neq i}}^{n} p_{i c}^{-}\left(1-u\left(k_{c}(t)\right)\right)\right] v_{i}\left(k-I_{i}, t\right)+ \\
+\sum_{\substack{j=1 \\
j \neq i}}^{n}\left[\mu_{j} p_{j i}^{+} u\left(k_{j}(t)\right) v_{i}\left(k+I_{i}-I_{j}, t\right)+\mu_{i} p_{i j}^{+} u\left(k_{i}(t)\right) v_{i}\left(k-I_{i}+I_{j}, t\right)+\mu_{i} p_{i j}^{-} v_{i}\left(k-I_{i}-I_{j}, t\right)\right]+
\end{gathered}
$$




$$
\begin{gathered}
+\sum_{\substack{c, s=1 \\
c, s \neq i, c \neq s}}^{n}\left[\mu_{s} p_{s c}^{+} u\left(k_{s}\right) v_{i}\left(k+I_{c}-I_{s}, t\right)+\mu_{c} p_{c s}^{-} u\left(k_{c}(t)\right) u\left(k_{s}(t)\right) v_{i}\left(k-I_{c}-I_{s}, t\right)\right]+(1) \\
+\lambda_{0 i}^{+} r_{0 i}\left(k+I_{i}, t\right)-\left[\mu_{i} p_{i 0} u\left(k_{i}(t)\right)+\lambda_{0 i}^{-} u\left(k_{i}(t)\right)+\mu_{i} \sum_{\substack{c=1 \\
c \neq i}}^{n} p_{i c}^{-}\left(1-u\left(k_{c}(t)\right)\right)\right] R_{i 0}\left(k-I_{i}, t\right)+ \\
+\sum_{\substack{j=1 \\
j \neq i}}^{n}\left[\mu_{j} p_{j i}^{+} u\left(k_{j}(t)\right) r_{i j}\left(k+I_{i}-I_{j}, t\right)-\mu_{i} p_{i j}^{+} u\left(k_{i}(t)\right) r_{j i}\left(k-I_{i}+I_{j}, t\right)-\right. \\
\left.-\mu_{i} p_{i j}^{-} u\left(k_{i}(t)\right) u\left(k_{j}(t)\right) r_{i j}\left(k-I_{i}-I_{j}, t\right)\right]
\end{gathered}
$$

\begin{tabular}{|c|c|c|}
\hline $\begin{array}{c}\text { Possible transitions } \\
\text { between network states }\end{array}$ & Transition probabilities & $\begin{array}{c}\text { Changes in the total volume } \\
\text { of claims in the system } S_{i} \text {, } \\
\text { associated with transitions } \\
\text { between network states }\end{array}$ \\
\hline$(k, t) \rightarrow(k, t+\Delta t)$ & $1-\sum_{j=1}^{n}\left[\lambda_{0 j}^{+}+\lambda_{0 j}^{-}+\mu_{j}\right] \Delta t+o(\Delta t)$ & $r_{i}(k) \Delta t+v_{i}(k, t)$ \\
\hline $\begin{array}{c}(k, t) \rightarrow\left(k+I_{j}, t+\Delta t\right) \\
j \neq i\end{array}$ & $\lambda_{0 j}^{+} \Delta t+o(\Delta t)$ & $r_{i}(k) \Delta t+v_{i}\left(k+I_{j}, t\right)$ \\
\hline $\begin{array}{c}(k, t) \rightarrow\left(k-I_{j}, t+\Delta t\right) \\
j \neq i\end{array}$ & $\begin{array}{l}\mu_{j} p_{j 0} u\left(k_{j}(t)\right) \Delta t+\lambda_{0 j}^{-} u\left(k_{j}(t)\right) \Delta t+ \\
+\mu_{j} \sum_{\substack{c=1 \\
c \neq i}}^{n} p_{j c}^{-}\left(1-u\left(k_{c}(t)\right)\right) \Delta t+o(\Delta t)\end{array}$ & $r_{i}(k) \Delta t+v_{i}\left(k-I_{j}, t\right)$ \\
\hline$(k, t) \rightarrow\left(k+I_{i}, t+\Delta t\right)$ & $\lambda_{0 i}^{+} \Delta t+o(\Delta t)$ & $r_{0 i}\left(k+I_{i}, t\right)+v_{i}\left(k+I_{i}, t\right)$ \\
\hline$(k, t) \rightarrow\left(k-I_{i}, t+\Delta t\right)$ & $\begin{array}{l}\mu_{i} p_{i 0} u\left(k_{i}(t)\right) \Delta t+\lambda_{0 i}^{-} u\left(k_{i}(t)\right) \Delta t+ \\
+\mu_{i} \sum_{\substack{c=1 \\
c \neq i}}^{n} p_{i c}^{-}\left(1-u\left(k_{c}(t)\right)\right) \Delta t+o(\Delta t)\end{array}$ & $\begin{array}{l}-R_{i 0}\left(k-I_{i}, t\right)+ \\
\quad+v_{i}\left(k-I_{i}, t\right)\end{array}$ \\
\hline $\begin{array}{c}(k, t) \rightarrow\left(k+I_{i}-I_{j}, t+\Delta t\right) \\
j \neq i\end{array}$ & $\mu_{j} p_{j i}^{+} u\left(k_{j}(t)\right) \Delta t+o(\Delta t)$ & $\begin{array}{l}r_{i j}\left(k+I_{i}-I_{j}, t\right)+ \\
+v_{i}\left(k+I_{i}-I_{j}, t\right)\end{array}$ \\
\hline $\begin{array}{c}(k, t) \rightarrow\left(k-I_{i}+I_{j}, t+\Delta t\right) \\
j \neq i\end{array}$ & $\mu_{i} p_{i j}^{+} u\left(k_{i}(t)\right) \Delta t+o(\Delta t)$ & $\begin{array}{l}-r_{j i}\left(k-I_{i}+I_{j}, t\right)+ \\
+v_{i}\left(k-I_{i}+I_{j}, t\right)\end{array}$ \\
\hline $\begin{array}{c}(k, t) \rightarrow\left(k+I_{c}-I_{s}, t+\Delta t\right) \\
c, s \neq i\end{array}$ & $\mu_{s} p_{s c}^{+} u\left(k_{s}(t)\right) \Delta t+o(\Delta t)$ & $\begin{array}{c}r_{i}(k) \Delta t+ \\
+v_{i}\left(k+I_{c}-I_{s}, t\right) \\
\end{array}$ \\
\hline $\begin{array}{c}(k, t) \rightarrow\left(k-I_{i}-I_{j}, t+\Delta t\right) \\
j \neq i\end{array}$ & $\mu_{i} p_{i j}^{-} u\left(k_{i}(t)\right) u\left(k_{j}(t)\right) \Delta t+o(\Delta t)$ & $\begin{array}{l}-r_{i j}\left(k-I_{i}-I_{j}, t\right)+ \\
+v_{i}\left(k-I_{i}-I_{j}, t\right)\end{array}$ \\
\hline $\begin{array}{c}(k, t) \rightarrow\left(k-I_{c}-I_{s}, t+\Delta t\right) \\
c, s \neq i\end{array}$ & $\mu_{c} p_{c s}^{-} u\left(k_{c}(t)\right) u\left(k_{s}(t)\right) \Delta t+o(\Delta t)$ & $\begin{array}{c}r_{i}(k) \Delta t+ \\
+v_{i}\left(k-I_{c}-I_{s}, t\right) \\
\end{array}$ \\
\hline
\end{tabular}

Table 1. Possible transitions between network states, their probabilities and changes in the volume of memory in the system 
The number of equations in this system is equal to the number of network states, i.e. for an open network is infinity. Formally, the system of equations (1) can be reduced to a system of countably many linear inhomogeneous ordinary differential equations with constant coefficients, which in matrix form can be written in the form

$$
\frac{d V_{i}(t)}{d t}=Q_{i}(t)+A V_{i}(t),
$$

where $V_{i}^{T}(t)=\left(v_{i}(1, t), v_{i}(2, t), \ldots, v_{i}(l, t), \ldots\right)$ - the required vector of the volume of the system's claims $S_{i}$. The solution of the system (2) can be found using the direct method (using the matrix exponent) [29]. Multiplying both sides of system (2) by $e^{-A t}$, we obtain

$$
V_{i}(t)=e^{A t} V_{i}(0)+\int_{0}^{t} e^{A(t-\tau)} Q_{i}(\tau) d \tau
$$

where: $e^{A t}=I+A t+\frac{A^{2} t^{2}}{2 !}+\ldots+\frac{A^{m} t^{m}}{m !}+\ldots$ - matrix exponent, $I$ - identity matrix. To find the matrix $e^{A t}$, as is known, we must find eigenvalues $q_{1}, q_{2}, \ldots, q_{l}, \ldots$ of the matrix $A$ and the complete system of corresponding right eigenvectors $u^{(1)}, u^{(2)}$, $\ldots, u^{(l)}, \ldots$, if possible. Then we have to use the representation $e^{A t}=U B(t) U^{-1}$, where $U$ - matrix whose columns are eigenvectors $u^{(1)}, u^{(2)}, \ldots, u^{(l)}, \ldots, B(t)$ - diagonal matrix:

$$
B(t)=\left(\begin{array}{ccccc}
e^{q_{1} t} & 0 & \cdots & 0 & \cdots \\
0 & e^{q_{2} t} & \cdots & 0 & \cdots \\
\cdots & \cdots & \cdots & \cdots & \cdots \\
0 & 0 & \cdots & e^{q_{l} t} & \cdots \\
& & \cdots & &
\end{array}\right) .
$$

But because of the unlimited dimension of the matrices $A, Q_{i}(t)$ in practice, this method can be used only in special cases when they have a special form.

\section{Analysis of expected volumes of claims in systems in the case when their changes from transitions between network states are random variables with given moments of the first two orders}

Let $\xi_{i}$ - the service time of the claim in the system $S_{i}$, distributed according to an exponential law with the distribution function $F_{\xi_{i}}(t)=1-e^{-\mu_{i} t}, i=\overline{1, n}$. Consider 
the dynamics of changes in the volume of claims in the system $S_{i}$. Suppose, at the initial instant of time, the volume of claims for this QS was equal to $v_{i 0}$. We will be interested in the total amount of claims in the system $V_{i}(t)$ at time $t$. Changing the volume of claims in the interval $[t, t+\Delta t)$ described by the relation

$$
V_{i}(t+\Delta t)=V_{i}(t)+\Delta V_{i}(t, \Delta t)
$$

where $\Delta V_{i}(t, \Delta t)$ - volume changes in QS $S_{i}$ on the time interval $[t, t+\Delta t), i=\overline{1, n}$.

To find the volume of claims in the system $S_{i}$, we write the conditional probabilities of events that can occur during the time $\Delta t$ and change in the volume of claims for this QS during this time. The following situations are possible:

1) with probability $\lambda_{0 i}^{+} \Delta t+o(\Delta t)$ to the system $S_{i}$ from the external environment will come positive claim, which will increase the volume of its claims by the amount $r_{0 i}$, where $r_{0 i}$ - random variable (RV) with mathematical expectation (E) $E\left\{r_{0 i}\right\}=a_{0 i}, i=\overline{1, n}$;

2) with probability $\lambda_{0 i}^{-} \Delta t+o(\Delta t)$ to the system $S_{i}$ from the external environment will come a negative claim, which will reduce the volume of its claims by the amount $-\bar{r}_{0 i}$, where $\bar{r}_{0 i}-\mathrm{RV}$ with $\mathrm{E} E\left\{\bar{r}_{0 i}\right\}=\bar{a}_{0 i}, i=\overline{1, n}$;

3) with probability $\mu_{i} p_{i 0} u\left(k_{i}(t)\right) \Delta t+o(\Delta t)$ a positive claim comes out from the network to the external environment, while the total amount of volume of claims $S_{i}$ is reduced by an amount which is equal to $R_{i 0}$, where $R_{i 0}-\mathrm{RV}$ with $\mathrm{E}$ $E\left\{R_{i 0}\right\}=b_{i 0}, i=\overline{1, n}$;

4) a positive claim from the system $S_{i}$ will transfer to the system $S_{j}$ with probability $\mu_{i} p_{i j}^{+} u\left(k_{i}(t)\right) \Delta t+o(\Delta t), i, j=\overline{1, n}, i \neq j$; with such a transition the volume of claims in the system $S_{i}$ decreases by an amount $R_{i j}\left(\xi_{i}\right)$, and the volume of claims of the system $S_{j}$ will increase by this amount:

$$
E\left\{R_{i j}\left(\xi_{i}\right)\right\}=\int_{0}^{\infty} R_{i j}(t) d F_{\xi_{i}}(t)=\mu_{i} \int_{0}^{\infty} R_{i j}(t) e^{-\mu_{i} t} d t=a_{i j}, i=\overline{1, n}, j=\overline{1, n}, i \neq j
$$

5) with probability $\mu_{j} p_{j i}^{+} u\left(k_{i}(t)\right) \Delta t+o(\Delta t)$ a positive claim will be transferred from the system $S_{j}$ to the system $S_{i}$, while the volume of claims in the system $S_{i}$ will increase by $R_{j i}\left(\xi_{j}\right)$, and the volume of claims in the system $S_{j}$ will decrease by this amount, $E\left\{R_{j i}\left(\xi_{j}\right)\right\}=a_{j i}, j=\overline{1, n}, j \neq i$;

6) a positive claim from the system $S_{i}$ will transfer to the system $S_{j}$ with probability $\mu_{i} p_{i j}{ }^{-} \Delta t+o(\Delta t)$ as a negative claim, $i, j=\overline{1, n}, i \neq j$; with such a transition, the volume of claims in the system $S_{i}$ increase by $\bar{R}_{i j}$, where $\bar{R}_{i j}-\mathrm{RV}$ with $\mathrm{E}$ $E\left\{\bar{R}_{i j}\right\}=c_{i j}, i, j=\overline{1, n}, i \neq j$; 
7) with probability $1-\sum_{j=1}^{n}\left(\lambda_{0 j}^{+}+\lambda_{0 j}^{-}+\mu_{j}\right) \Delta t+o(\Delta t)$ on time $\Delta t$ the network state will not change;

8) for every short period of time $\Delta t$ the system $S_{i}$ because of the presence of claims in it increases its volume of claims by the amount $r_{i} \Delta t$, where $r_{i}-\mathrm{RV}$ with $\mathrm{E} E\left\{r_{i}\right\}=d_{i}, i=\overline{1, n}$.

It follows from the above:

$$
\Delta V_{i}(t, \Delta t)=\left\{\begin{array}{l}
\quad \\
r_{0 i}+r_{i} \Delta t \text { with pr. } \lambda_{0 i}^{+} \Delta t+o(\Delta t), \\
-\bar{r}_{0 i}+r_{i} \Delta t \text { with pr. } \lambda_{0 i}^{-} \Delta t+o(\Delta t), \\
-R_{i 0}+r_{i} \Delta t \text { with pr. } \mu_{i} p_{i 0} u\left(k_{i}(t)\right) \Delta t+o(\Delta t), \\
-R_{i j}\left(\xi_{i}\right)+r_{i} \Delta t \text { with pr. } \mu_{i} p_{i j}^{+} u\left(k_{i}(t)\right) \Delta t+o(\Delta t), j=\overline{1, n}, j \neq i, \\
R_{j i}\left(\xi_{j}\right)+r_{i} \Delta t \text { with pr. } \mu_{j} p_{j i}^{+} u\left(k_{j}(t)\right) \Delta t+o(\Delta t), j=\overline{1, n}, j \neq i, \\
-\bar{R}_{j i}+r_{i} \Delta t \text { with pr. } \mu_{i} p_{i j}^{-} \Delta t+o(\Delta t), j=\overline{1, n}, j \neq i, \\
r_{i} \Delta t \text { with pr. } 1-\sum_{j=1}^{n}\left[\lambda_{0 j}^{+}+\lambda_{0 j}^{-}+\mu_{j}\right] \Delta t+o(\Delta t) .
\end{array}\right.
$$

Let's find the expression for changing the average volume of applications in the system $S_{i}$ at the time $t$. Suppose that all network systems operate in a high load mode, i.e., $k_{i}(t)>0, \forall t>0, i=\overline{1, n}$. Then, taking (5) into account for the mathematical expectation, we can write:

$$
\begin{gathered}
E\left\{\Delta V_{i}(t, \Delta t)\right\}=\left(a_{0 i}+d_{i} \Delta t\right)\left(\lambda_{0 i}^{+} \Delta t+o(\Delta t)\right)+\left(-\bar{a}_{0 i}+d_{i} \Delta t\right)\left(\lambda_{0 i}^{-} \Delta t+o(\Delta t)\right)+ \\
+\left(-b_{i 0}+d_{i} \Delta t\right)\left(\mu_{i} p_{i 0} \Delta t+o(\Delta t)\right)+ \\
+\sum_{j=1}^{n}\left[\left(-a_{i j}+d_{i} \Delta t\right)\left(\mu_{i} p_{i j}^{+} \Delta t+o(\Delta t)\right)\right]+\sum_{j=1}^{n}\left[\left(a_{j i}+d_{i} \Delta t\right)\left(\mu_{j} p_{j i}^{+} \Delta t+o(\Delta t)\right)\right]+ \\
+\sum_{j=1}^{n}\left[\left(-c_{i j}+d_{i} \Delta t\right)\left(\mu_{i} p_{i j}^{-} \Delta t+o(\Delta t)\right)\right]+d_{i} \Delta t\left(1-\sum_{j=1}^{n}\left[\lambda_{0 j}^{+}+\lambda_{0 j}^{-}+\mu_{j}\right] \Delta t+o(\Delta t)\right)= \\
=\left[a_{0 i} \lambda_{0 i}^{+}-\bar{a}_{0 i} \lambda_{0 i}^{-}-b_{i 0} \mu_{i} p_{i 0}\right] \Delta t+ \\
+\sum_{j=1}^{n}\left[-a_{i j} \mu_{i} p_{i j}^{+}+a_{j i} \mu_{j} p_{j i}^{+}-c_{i j} \mu_{i} p_{i j}^{-}+d_{i}\right] \Delta t+o(\Delta t), i=\overline{1, n}
\end{gathered}
$$


Therefore, as follows from (4),

$$
\begin{gathered}
V_{i}(t+\Delta t)=M\left\{V_{i}(t+\Delta t)\right\}=v_{i}(t)= \\
=\left(a_{0 i} \lambda_{0 i}^{+}-\bar{a}_{0 i} \lambda_{0 i}^{-}-\mu_{i} \sum_{j=1}^{n} c_{i j} p_{i j}^{-}+d_{i}-b_{i 0} \mu_{i} p_{i 0}-\sum_{j=1}^{n} a_{i j} \mu_{i} p_{i j}^{+}+\sum_{j=1}^{n} a_{j i} \mu_{j} p_{j i}^{+}\right) \Delta t+o(\Delta t),
\end{gathered}
$$

i.e.

$v_{i}(t)=v_{i 0}+\left[\lambda_{0 i}^{+} a_{0 i}-\lambda_{0 i}^{-} \bar{a}_{0 i}-\mu_{i}\left(b_{i 0} p_{i 0}+\sum_{j=1}^{n}\left(a_{i j} p_{i j}^{+}+c_{i j} p_{i j}^{-}\right)\right)+\sum_{j=1}^{n} \mu_{j} a_{j i} p_{j i}^{+}+d_{i}\right] t, i=\overline{1, n}$.

\section{Example}

Let the number of QS in the network $n=10$. Intensity of the input flow of positive and negative claims $\lambda_{0 i}^{+}$and $\lambda_{0 i}^{-}$are equal respectively $\lambda_{01}^{+}=2, \lambda_{04}^{+}=4, \lambda_{07}^{+}=3$, $\lambda_{01}^{-}=1, \lambda_{04}^{-}=2, \lambda_{07}^{-}=3$, the rest are zero. Intensity of service of claims $\mu_{i}$ are equal $\mu_{1}=\mu_{2}=\mu_{3}=2, \mu_{4}=1, \mu_{5}=3, \mu_{6}=5, \mu_{7}=3, \mu_{8}=13, \mu_{9}=7, \mu_{10}=8$. Let the probabilities $p_{i j}^{+}$are equal respectively $p_{12}^{+}=p_{13}^{+}=\frac{1}{8}, p_{21}^{+}=p_{23}^{+}=p_{24}^{+}=p_{25}^{+}=p_{31}^{+}=$ $=p_{32}^{+}=p_{36}^{+}=p_{37}^{+}=0.1, \quad p_{42}^{+}=p_{45}^{+}=p_{48}^{+}=p_{52}^{+}=p_{54}^{+}=p_{56}^{+}=p_{58}^{+}=p_{63}^{+}=p_{65}^{+}=p_{67}^{+}=$ $=p_{69}^{+}=p_{73}^{+}=p_{76}^{+}=p_{79}^{+}=p_{84}^{+}=\frac{1}{8} ; \quad p_{85}^{+}=p_{89}^{+}=p_{8,10}^{+}=p_{96}^{+}=p_{97}^{+}=p_{98}^{+}=p_{9,10}^{+}=\frac{1}{8}$, $p_{10,8}^{+}=p_{10,9}^{+}=0.2$, the rest are zero. The probabilities that positive claims served in the QS $S_{i}$, sent to the QS $S_{j}$ as negative claims are equal $p_{12}^{-}=p_{13}^{-}=p_{42}^{-}=p_{45}^{-}=p_{48}^{-}=$ $=p_{52}^{-}=p_{54}^{-}=p_{56}^{-}=p_{58}^{-}=p_{63}^{-}=p_{65}^{-}=p_{67}^{-}=p_{69}^{-}=p_{73}^{-}=p_{79}^{-}=p_{84}^{-}=p_{85}^{-}=p_{89}^{-}=p_{8,10}^{-}=p_{96}^{-}=$ $=p_{97}^{-}=p_{98}^{-}=p_{9,10}^{-}=\frac{1}{9}, \quad p_{21}^{-}=p_{23}^{-}=p_{24}^{-}=p_{25}^{-}=p_{31}^{-}=p_{32}^{-}=p_{36}^{-}=p_{37}^{-}=p_{10,8}^{-}=p_{10,9}^{-}=\frac{1}{11}$, the rest are zero. Probabilities of exiting claims from the network to the external environment $p_{40}=p_{70}=\frac{7}{24}, p_{10,0}=\frac{4}{15}$. The corresponding mathematical expectations are equal: $a_{01}=a_{05}=a_{06}=a_{010}=\bar{a}_{03}=10000, a_{02}=20000, a_{03}=a_{08}=30000$, $\bar{a}_{01}=1000, a_{04}=a_{09}=\bar{a}_{05}=50000, a_{07}=40000, \bar{a}_{02}=60000, \bar{a}_{04}=25000, \bar{a}_{06}=7000$, $\bar{a}_{07}=3000, \quad \bar{a}_{08}=2500, \quad \bar{a}_{09}=1200, \bar{a}_{010}=9000, b_{10}=b_{50}=b_{60}=b_{70}=b_{10,0}=c_{12}=$ $b_{10}=b_{50}=b_{60}=b_{70}=b_{10,0}=c_{12}=c_{31}=c_{32}=c_{36}=c_{37}=1000, c_{63}=c_{65}=c_{67}=c_{59}=1700$, $c_{73}=c_{76}=c_{79}=2300, \quad b_{20}=c_{21}=c_{23}=c_{24}=c_{25}=c_{45}=c_{48}=c_{84}=c_{85}=c_{89}=c_{8,10}=$ $=2000, d_{1}=100, d_{8}=100, d_{5}=200, b_{40}=b_{90}=5000, b_{30}=b_{80}=c_{96}=c_{97}=c_{98}=$ $=c_{9,10}=3000, c_{31}=c_{52}=c_{54}=c_{56}=c_{58}=1500, c_{10,8}=c_{10,9}=1300, d_{2}=200, d_{3}=300$, 
$d_{4}=120, d_{7}=800, d_{9}=120$, the rest are zero. Random changes in the volume of claims in the system $S_{i}$ has the form:

$$
\begin{aligned}
& R_{12}\left(\xi_{1}\right)=3000 \xi_{1}, \quad R_{21}\left(\xi_{2}\right)=2500+\xi_{2}, \quad R_{23}\left(\xi_{2}\right)=100 \xi_{2}, \quad R_{24}\left(\xi_{2}\right)=200 \xi_{2}, \\
& R_{25}\left(\xi_{2}\right)=2000 \xi_{2}, \quad R_{31}\left(\xi_{3}\right)=0,5 \xi_{3}+1000, \quad R_{32}\left(\xi_{3}\right)=0,1 \xi_{3}+10000 \text {, } \\
& R_{36}\left(\xi_{3}\right)=0,5 \xi_{3}+450, \quad R_{37}\left(\xi_{3}\right)=0,5 \xi_{3}+5000, \quad R_{42}\left(\xi_{4}\right)=10 \xi_{4}-100 \text {, } \\
& R_{8,10}\left(\xi_{8}\right)=\xi_{8}\left(\xi_{8}+200\right), \quad R_{45}\left(\xi_{4}\right)=\left(\xi_{4}+100\right) \xi_{4}, \quad R_{48}\left(\xi_{4}\right)=2000 \xi_{4} \text {, } \\
& R_{52}\left(\xi_{5}\right)=1000 \xi_{5}-10, \quad R_{96}\left(\xi_{9}\right)=\xi_{9}+1000, \quad R_{54}\left(\xi_{5}\right)=1000 \xi_{5}+100 \text {, } \\
& R_{56}\left(\xi_{5}\right)=1000 \xi_{5}+300, R_{58}\left(\xi_{5}\right)=1000 \xi_{5}, R_{89}\left(\xi_{8}\right)=\xi_{8}\left(\xi_{8}-10\right), R_{63}\left(\xi_{6}\right)=\xi_{6}+1000 \text {, } \\
& R_{65}\left(\xi_{5}\right)=5 \xi_{5}+1000, \quad R_{67}\left(\xi_{6}\right)=3 \xi_{6}+1000, \quad R_{10,8}\left(\xi_{10}\right)=3000 \xi_{10}, \\
& R_{69}\left(\xi_{6}\right)=2 \xi_{6}+1000, \quad R_{73}\left(\xi_{7}\right)=1000 \xi_{7}-100, \quad R_{76}\left(\xi_{7}\right)=1000 \xi_{7} \text {, } \\
& R_{10,9}\left(\xi_{10}\right)=3000 \xi_{10}-100, \quad R_{79}\left(\xi_{7}\right)=100 \xi_{7}+300, \quad R_{84}\left(\xi_{8}\right)=\xi_{8}\left(\xi_{8}+1000\right) \text {, } \\
& R_{85}\left(\xi_{8}\right)=\xi_{8}\left(\xi_{8}+100\right), \quad R_{97}\left(\xi_{9}\right)=3 \xi_{9}+1000, \quad R_{98}\left(\xi_{9}\right)=10 \xi_{9}+1000 \text {, }
\end{aligned}
$$

$R_{9,10}\left(\xi_{9}\right)=\xi_{9}-100$.

The mathematical expectations of these random volumes were calculated in the Mathematica package and are equal, respectively

$$
\begin{array}{llrl}
a_{12}=144000 e^{-2 t} t, \quad a_{13}=48 e^{-2 t}(0,1+1000 t), & a_{21}=48 e^{-2 t}(2500+t), & a_{23}=4800 e^{-2 t} t, \\
a_{24}=9600 e^{-2 t} t, a_{25}=96000 e^{-2 t} t, a_{31}=48 e^{-2 t}(1000+0,5 t), & a_{32}=48 e^{-2 t}(1000+0,1 t), \\
a_{36}=48 e^{-2 t}(450+0,5 t), & a_{42}=240 e^{-t}(t-10), \quad a_{45}=24 e^{-t}(t+100), & a_{37}=48000 e^{-t}, \\
a_{52} & =720 e^{-3 t}(100 t-1), & a_{54}=7200 e^{-3 t}(10 t-1), & a_{56}=7200 e^{-3 t}(10 t+3), \\
a_{58}=72000 e^{-3 t}, \quad a_{96}=576000 e^{-8 t} t, \quad a_{63}=96 e^{-4 t}(t+1000), & a_{65}=96 e^{-4 t}(5 t+1000), \\
a_{67}=96 e^{-4 t}(3 t+1000), & a_{76}=72000 t e^{-3 t}, & a_{69}=96 e^{-4 t}(2 t+1000), \\
a_{73}=7200 e^{-3 t}(-1+1000 t), & a_{79}=7200 e^{-3 t}(3+t), & a_{84}=312 e^{-13 t}(1000+t), \\
a_{89}=312 e^{-13 t}(-10+t) t, & a_{8,10}=312 e^{-13 t}(200+t), & a_{96}=168 e^{-7 t}(1000+t), \\
a_{85}=312 e^{-13 t}(100+t), & a_{97}=168 e^{-7 t}(1000+3 t), & a_{98}=1680 e^{-7 t}(100+t), \\
a_{9,10}=168 e^{-7 t}(t-100) . & &
\end{array}
$$

Let the volume of claims at the initial instant of time be $v_{i 0}=0, i=\overline{1, n}$. Consider a time interval of length in $24 \mathrm{~h}, t \in[0, T], T=24$.

Finding the expected volume of claims in the network systems was implemented as a program for a package of mathematical calculations Wolfram Mathematica. Figure 1 shows the change in the expected volume of claims in the system for HM-network with negative claims. 


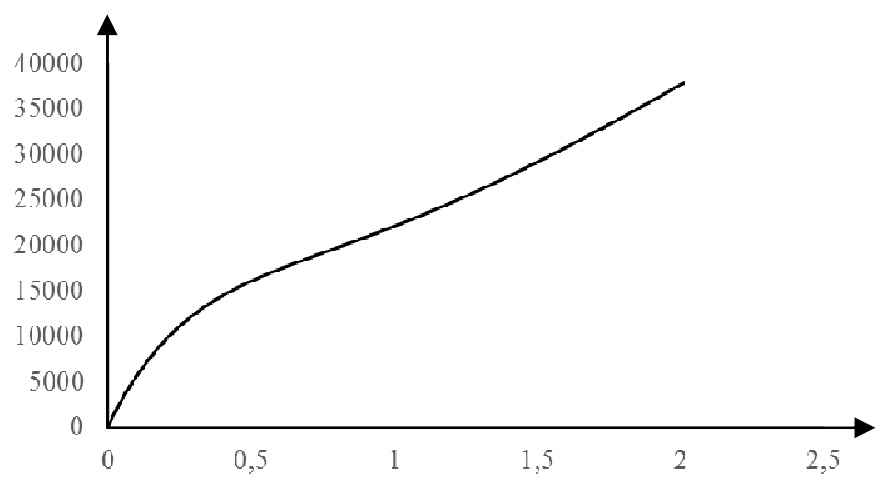

Fig. 1. Volume changes of claims in the $i$-th QS

\section{Conclusions}

Further researching in this direction can be associated with the analysis of arbitrary (non-Markov) networks with claims with random volume and Markov networks with various other features.

\section{References}

[1] Tikhonenko, O. (2006). Metody probalistycznne analizy systemów informacyjnych. Warszawa: Akademicka Oficyna Wydawnicza EXIT.

[2] Matalytski, M., Tikhonenko, O., \& Koluzaeva, E. (2011). Systems and queueing networks: analysis and application [In Russian: Sistemy i seti massovogo obsluzhivania: analiz i primenenija]. Grodno; GrSU.

[3] Bufferbloat [Electronic resource]: Wikipedia: http: // en. wikipedia. org /wiki/

[4] Matalytski, M., \& Naumenko, V. (2016). Stochastic networks with nonstandard moving customers [In Russian: Stokhasticheskie seti s nestandartnym peremeshcheniem zaiavok]. Grodno; GrSU.

[5] Matalytski, M., \& Naumenko, V. (2015). Zastosowanie HM-sieci kolejkowych dla wyznaczenia objętości pamięci systemów informacyjnych. Studia Informatica, 35(3), 63-69.

[6] Matalytski, M., \& Zając, P. (2015). Finding of expected volumes of request in systems of the queueing network with limited amount of spaces of expectations. Studia Informatica, 38(1), 89-104.

[7] Zając, P., \& Matalytski, M. (2017). Expected volumes of requests in systems of the queueing network with a limited number of waiting places. Scientific Issues Jan Dtugosz University in Częstochowa Mathematics XXII, 141-160.

[8] Matalytski, M., \& Zajac, P. (2017). Application of HM-networks with impatient claims in finding the memory capacity in the information systems. Journal of Applied Mathematics and Computational Mechanics, 16(3), 47-59.

[9] Matalytski, M., Zajac, P., \& Kopats, D. (2018). Application of HM-networks with unreliable systems for finding the memory capacity in the information systems. Journal of Applied Mathematics and Computational Mechanics, 17(2).

[10] Gelenbe, E. (1991). Product form queueing networks with negative and positive customers. Journal of Applied Probability, 28, 656-663. 
[11] Gelenbe, E., Glynn, P., \& Sigman, K. (1991). Queues with negative customers. Journal of Applied Probability, 28, 242-250.

[12] Gelenbe, E., \& Schassberger, R. (1992). Stability of G-networks. Probability and its Applications in Engineering and the Information Sciences. Cambridge University Press. 6, 271-276.

[13] Shanguin, V. (2013). Information security of computer systems and networks. M.: Vocational Education. Infra-M., 416.

[14] Schneier, B. (2000). Secrets and Lies. Data Security in the Digital World. St. Petersburg.: Petersburg, 432.

[15] Bocharov, P.P., \& Vishnevsky, V.M. (2003). G-networks: development of the theory of multiplicative networks. Automation and Telemechanics, 5, 46-74.

[16] Gelenbe, E. (1994). G-networks: a unifying model for neural and queueing networks. Annals of Operations Research, 48, 433-461.

[17] Gelenbe, E. (1989). Random neural Networks with negative and positive signals and product form solution. Neural Computation, 1, 502-510.

[18] Kim, H., \& Gelenbe, E. (2013). G-networks towards synthetic biology. A brief review. Conf Proc. IEEE Eng. Med. Biol. Soc. 1, 579-583.

[19] Artalejo, A., \& Jesus, R. (2000). G-networks: A versatile approach for work removal in queueing networks. European Journal of Operational Research, 126, 233-249.

[20] Artalejo, J.R. (1996). Retrial queues with negative arrivals. Proceedings of International Conference on Stochastic Processes. Cochin, 159-168.

[21] Boucherie, R.J., \& van Dijk, N.M. (1994). Local balance in queueing networks with positive and negative customers. Annals of Operations Research, 48, 463-492.

[22] Chao, X., \& Zheng, S. (1998). A result on networks of queues with customer coalescence and state-dependent signalling. Journal of Applied Probability, 35, 151-164.

[23] Chao, X. (1995). Networks of queues with customers, signals and arbitrary service time distributions. Operations Research, 43, 537-550.

[24] Fourneau, J.M., Gelenbe, E., \& Surosc, R. (1996). G-networks with multiple classes of negative and positive customers. Theoretical Computer Science, 155, 1, 141-156.

[25] Gelenbe, E. (2002). G-networks: Multiple classes of positive customers, signals, and product form. Results performance evaluation of complex systems: Techniques and Tools. Lecture Notes in Computer Science. 1-16.

[26] Gelenbe, E. (1993). G-networks with instantaneous customer movement. Journal of Applied Probability, 30, 742-748.

[27] Gelenbe, E., \& Labed, A. (1998). G-networks with multiple classes of signals and positive customers. European Journal of Operational Research, 108, 293-305.

[28] Gelenbe, E. \& Fourneau, J.M. (2002). G-Networks with resets. Performance Evaluation, 49, 179-192.

[29] Gantmakher, F.R. (1988). Theory of Matrices. M.: Science, 547. 